\title{
Geometry and transport in a model of two coupled quadratic nonlinear waveguides
}

Stirling, James R.; Bang, Ole; Christiansen, Peter Leth; Zakynthianaki, Maria S.; Johansen, Steffen Kjær

\section{Published in:}

Chaos

Link to article, DOI:

$10.1063 / 1.2840461$

Publication date:

2008

Document Version

Publisher's PDF, also known as Version of record

Link back to DTU Orbit

Citation (APA):

Stirling, J. R., Bang, O., Christiansen, P. L., Zakynthianaki, M. S., \& Johansen, S. K. (2008). Geometry and transport in a model of two coupled quadratic nonlinear waveguides. Chaos, 18(1), 013116.

https://doi.org/10.1063/1.2840461

\section{General rights}

Copyright and moral rights for the publications made accessible in the public portal are retained by the authors and/or other copyright owners and it is a condition of accessing publications that users recognise and abide by the legal requirements associated with these rights.

- Users may download and print one copy of any publication from the public portal for the purpose of private study or research.

- You may not further distribute the material or use it for any profit-making activity or commercial gain

- You may freely distribute the URL identifying the publication in the public portal 


\title{
Geometry and transport in a model of two coupled quadratic nonlinear waveguides
}

\author{
James R. Stirling, ${ }^{1, a)}$ Ole Bang, ${ }^{2}$ Peter L. Christiansen, ${ }^{3}$ Maria S. Zakynthinaki, ${ }^{4, b)}$ \\ and Steffen Kjær Johansen ${ }^{5}$ \\ ${ }_{1}^{1}$ Facultad de Ciencias de la Actividad Física y del Deporte, Universidad Politécnica de Madrid, \\ Avda Martin Fierro s/n, 28040 Madrid, Spain \\ ${ }^{2} \mathrm{COM} \cdot \mathrm{DTU}$ Department of Communications, Optics and Materials, Technical University of Denmark, \\ DK-2800 Kongens Lyngby, Denmark \\ ${ }^{3}$ Informatics and Mathematical Modelling and Department of Physics, Technical University of Denmark, \\ DK-2800 Kongens Lyngby, Denmark \\ ${ }^{4}$ Instituto de Ciencias Matemáticas CSIC-UAM-UC3M-UCM c/ Serrano 121, 28006 Madrid, Spain \\ ${ }^{5}$ Woven Reality ApS, Diplomvej 373, DK-2800 Kongens Lyngby, Denmark
}

(Received 12 October 2007; accepted 16 January 2008; published online 10 March 2008)

\begin{abstract}
This paper applies geometric methods developed to understand chaos and transport in Hamiltonian systems to the study of power distribution in nonlinear waveguide arrays. The specific case of two linearly coupled $\chi^{(2)}$ waveguides is modeled and analyzed in terms of transport and geometry in the phase space. This gives us a transport problem in the phase space resulting from the coupling of the two Hamiltonian systems for each waveguide. In particular, the effect of the presence of partial and complete barriers in the phase space on the transfer of intensity between the waveguides is studied, given a specific input and range of material properties. We show how these barriers break down as the coupling between the waveguides is increased and what the role of resonances in the phase space has in this. We also show how an increase in the coupling can lead to chaos and global transport and what effect this has on the intensity. (C) 2008 American Institute of Physics.
\end{abstract}

[DOI: $10.1063 / 1.2840461]$

We first present the general physical model for an arbitrary number of quadratic nonlinear waveguides, coupled linearly to each other. We normalize the system so that the dynamical evolution variable, i.e., the distance along the waveguides, can be big and therefore considered as a time variable, as is conventional in transport and chaos studies. We then focus on the two-core coupler (two waveguides) and investigate how structures in the phase space can lead to information about the coupling required for complete transfer of intensity from the fundamental wave (FW) in one waveguide to the second harmonic (SH; at twice the frequency) in the other waveguide. In particular, we investigate the structures that act as partial or complete barriers in the phase space. We study how these structures are destroyed as the coupling between the two waveguides is increased from the uncoupled regime to a critical value and beyond. The present work also includes a study of the effect of resonances in the phase space, as well as their connection with the destruction of complete barriers to transport. The sensitivity of the resulting intensity distribution to the strength of the coupling between the FW in the two waveguides $(\rho)$ is also studied. In particular, we show how the nature of the structures (or barriers) in the phase space can be changed with small changes in $\rho$, such

\footnotetext{
${ }^{a)}$ Electronic mail: jr.stirling@upm.es.

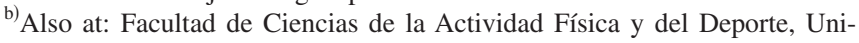
versidad Politécnica de Madrid, Avda Martin Fierro s/n, 28040 Madrid, Spain.
}

that a very ordered output can change to an output which appears ordered over a long time, but then suddenly becomes disordered or more complicated, to finally reach a very disordered state appearing to be the product of spatial-temporal chaos. Such behavior is of much practical importance, as it shows that, for certain values of $\rho$, a small change in $\rho$ can produce very different outputs. This also shows that such sensitivity is inherent in the system and is not a product of noise or experimental error.

\section{INTRODUCTION}

In this paper we study the geometry of the phase space and the resulting transport and chaotic behavior in a fundamental discrete quadratic nonlinear system of equations, which is used in solid-state physics to describe nonlinear interface waves between two media close to Fermi resonance, ${ }^{1,2}$ and in optics to describe arrays of waveguides with a quadratic (or $\chi^{(2)}$ ) nonlinearity. ${ }^{3-5}$ Here we focus on the applications in nonlinear optics.

In particular we use tools from dynamical systems to understand the geometry, chaos, and transport in the phase space with the aim of applying our findings to the understanding of the power distribution in quadratic nonlinear waveguide arrays. The specific case of two coupled $\chi^{(2)}$ waveguides is modeled (see Fig. 1) and analyzed in terms of transport and geometry in the phase space.

Propagation in quadratic nonlinear materials is characterized by the nonlinear coupling between a wave at the fun- 


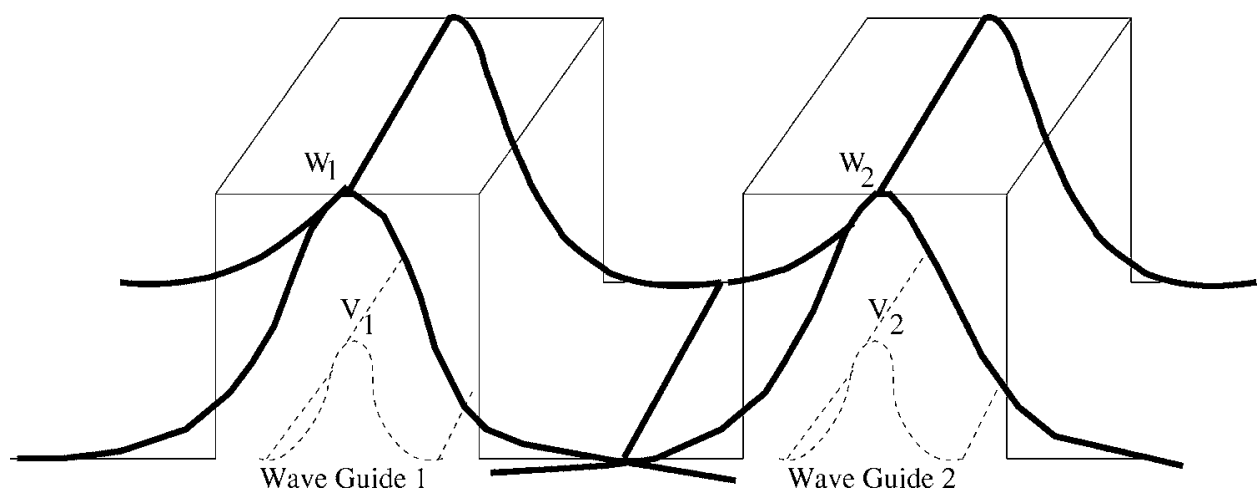

FIG. 1. The coupled waveguide system with fundamental waves $W_{1,2}$ and second-harmonic waves $V_{1,2}$.

damental frequency and a wave at the double secondharmonic frequency. Between neighboring waveguides only the fundamental waves are assumed to couple linearly to each other (see Fig. 2). This is a reasonable approximation because the second-harmonic modes are typically much better confined to the waveguide than the FWs, creating a negligible overlap between the second harmonic modes in neighboring waveguides (see Fig. 1). For more details regarding the physics of the problem, see Refs. 5 and 4.

Mathematically speaking, the system under study gives us a transport problem in a four degree of freedom Hamiltonian system. Geometry, transport, and chaos in Hamiltonian systems is a rich area of dynamical systems in which many tools have been developed. ${ }^{6-18}$ It should be noted that Arnold diffusion, or global instability, ${ }^{19-21}$ can take place in our system, since it possesses enough degrees of freedom. The effect of the presence of partial and complete barriers in the phase space and their effect on transport has many applications. Geometric methods related to transport in the phase space of Hamiltonian systems have been used to understand the dynamics occurring in many different applications. For example, there has been much work on this area in fluid dynamics related to the problems of mixing and chaotic advection in laminar flows (for a review, see Ref. 22) and also pollution dynamics in estuarine flows. ${ }^{23-25}$ However, the application of such methods to nonlinear optics and coupled nonlinear waves guides is new. In what follows, we show

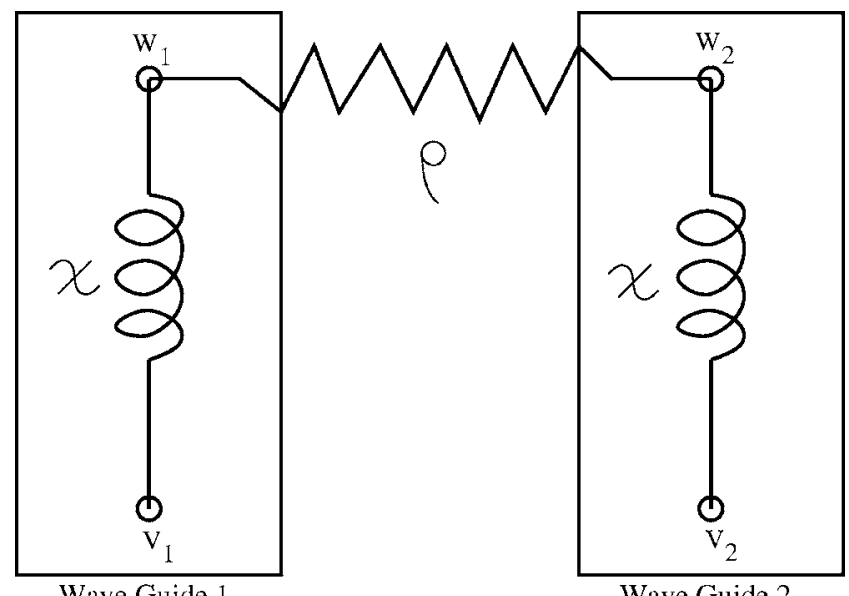

Wave Guide 1

Wave Guide 2

FIG. 2. Spring system corresponding to the model of the two coupled waveguides. $\rho$ is a linear spring and $\chi$ is a nonlinear spring. how the geometry and transport in the phase space affects the transfer of intensity between the waveguides, given a specific input and a range of material properties.

In the following sections, we first introduce the physical system and the dynamical equations. We then provide an analysis of the stationary solutions, the fixed points and their stability. We then go on to study the barriers to transport in the phase space and what effect they have on the transfer of intensity between the waveguides.

\section{PHYSICAL SYSTEM AND NORMALIZATION}

In a single waveguide, the fundamental wave (FW) with frequency $\omega$ is propagating along the waveguide in the $z$ direction, while being nonlinearly coupled to its second harmonic $(\mathrm{SH})$ at twice the frequency $\omega$. The evolution of the envelope of the FW, i.e., $E_{1, n}(z)$, and the second harmonic, i.e., $E_{2, n}(z)$, in waveguide $n$ is governed by the equations

$$
\begin{aligned}
& i \partial_{z} E_{1, n}+\rho_{1}\left(E_{1, n+1}+E_{1, n-1}\right)+\chi_{1} E_{1, n}^{*} E_{2, n} e^{-i \Delta k z}=0, \\
& i \partial_{z} E_{2, n}+\rho_{2}\left(E_{2, n+1}+E_{2, n-1}\right)+\chi_{2} E_{1, n}^{2} e^{i \Delta k z}=0 .
\end{aligned}
$$

Here, $\Delta k$ is the phase mismatch between the $\mathrm{FW}$ and the $\mathrm{SH}$, $\rho_{1,2}$ are coupling coefficients determining the strength of the coupling between the fields in adjacent waveguides, and $\chi_{1,2}$ are the strengths of the nonlinearity. We let $L$ be the length of the waveguide array with $z \in[0, L]$ and $n_{0}$ the total number of waveguides with $n=1, \ldots, n_{0}$. For this system the total intensity

$$
I=\frac{1}{2} \eta_{0} \sum_{n}\left(N_{1}\left|E_{1, n}\right|^{2}+N_{2}\left|E_{2, n}\right|^{2}\right)
$$

as well as the Hamiltonian are conserved quantities. In Eq. (3), $N_{1,2}$ are the linear refractive indices at the two frequencies, respectively, and $\eta_{0}$ is the specific admittance of vacuum.

We assume that $\rho_{2}=0$ since $E_{2, n}$ is always more strongly confined transversely in a waveguide than $E_{1, n}$. We introduce the dimensionless propagation variable $\tau=z / l_{c}$, where $l_{c}$ $=\pi / \Delta k$ is the so-called coherence length. This means that the dimensionless mismatch parameter is fixed to $\beta=\Delta k l_{c}=\pi$. In typical experiments, the coherence length is much smaller than the length of the waveguide, which means that the dynamical evolution variable $\tau \in\left[0, L / l_{c}\right]$ can be very large and treated as a time coordinate, the typical coordinate used in studies of transport and chaos in Hamiltonian systems. 
We further normalize the fields as $E_{1, n}(z)=a_{1} W_{n}(\tau)$ and $E_{2, n}(z)=a_{2} V_{n}(\tau) e^{i \beta \tau}$. We choose $a_{n}=\sqrt{2 I /\left(N_{n} \eta_{0}\right)}$ in order to obtain the nice property that the normalized total intensity is 1 :

$$
\sum_{n}\left(\left|W_{n}\right|^{2}+\left|V_{n}\right|^{2}\right)=1
$$

Finally, we neglect linear dispersion, so that $N_{1}=N_{2}$, and nonlinear dispersion, so that $\chi_{1}=\chi_{2}$. The resulting coupled equations governing the propagation of the normalized field components are

$$
\begin{aligned}
& i \dot{W}_{n}+\rho\left(W_{n+1}+W_{n-1}\right)+\chi W_{n}^{*} V_{n}=0, \\
& i \dot{V}_{n}-\beta V_{n}+\chi W_{n}^{2}=0,
\end{aligned}
$$

where $\rho=\rho_{1} l_{c}$ is the normalized FW coupling coefficient and $\chi=\chi_{1} l_{c} a_{1}$ is the normalized quadratic nonlinearity.

The Hamiltonian of the system takes the form

$H=\sum_{n=1}^{n_{0}} \beta\left|V_{n}\right|^{2}-2 \rho W_{n}^{*}\left(W_{n+1}+W_{n-1}\right)-2 \chi \operatorname{Re}\left\{W_{n}^{2} V_{n}^{*}\right\}$

and is a conserved quantity; i.e., $\dot{H}=0$. In the following, we keep $\beta=\pi$ and consider what happens when we increase $\tau$ from 0 to some large but finite value $\tau_{0}=L / l_{c}$, while $\rho$ and $\chi$ are varied over a range of real values.

\section{A. Equations for two-core coupler}

We shall restrict our investigation to the so-called twocore coupler (the dimer), where we have only two waveguides (i.e., $n=1,2)$, which are either coupled or uncoupled. The system of equations (5)-(7) then reduces to the following set of coupled ordinary differential equations:

$$
\begin{aligned}
& i \dot{W}_{1}+\rho W_{2}+\chi W_{1}^{*} V_{1}=0, \\
& i \dot{V}_{1}-\beta V_{1}+\chi W_{1}^{2}=0, \\
& i \dot{W}_{2}+\rho W_{1}+\chi W_{2}^{*} V_{2}=0, \\
& i \dot{V}_{2}-\beta V_{2}+\chi W_{2}^{2}=0,
\end{aligned}
$$

with the condition that

$$
\left|W_{1}\right|^{2}+\left|V_{1}\right|^{2}+\left|W_{2}\right|^{2}+\left|V_{2}\right|^{2}=1,
$$

and the Hamiltonian

$$
\begin{aligned}
H= & \beta\left(\left|V_{1}\right|^{2}+\left|V_{2}\right|^{2}\right)-2 \chi\left(\operatorname{Re}\left\{W_{1}^{2} V_{1}^{*}\right\}+\operatorname{Re}\left\{W_{2}^{2} V_{2}^{*}\right\}\right) \\
& -2 \rho\left(W_{1}^{*} W_{2}+W_{2}^{*} W_{1}\right) .
\end{aligned}
$$

Separating into real $\left(W_{n R}, V_{n R}\right)$ and imaginary $\left(W_{n I}, V_{n I}\right)$ terms gives eight coupled ordinary differential equations, which describe the dynamics in the phase space for the dimer with $\rho \neq 0$. The equations for the monomer with no coupling between the waveguides can be obtained by setting $\rho=0$.

\section{STATIONARY SOLUTIONS, FIXED POINTS, AND STABILITY}

In the following, we look for solutions to the equations set up in the previous section and we study their stability. In particular, we look for stationary solutions, which are solutions for which all individual intensities, i.e., $\left|W_{n}\right|^{2}$ and $\left|V_{n}\right|^{2}$, are constant. Stationary solutions have the general form $W_{n}(\tau)=w_{n} e^{i \lambda \tau}$ and $V_{n}(\tau)=v_{n} e^{i 2 \lambda \tau}$, where the amplitudes $w_{n}$ and $v_{n}$ are constants and the solution parameter $\lambda$ is a real constant. Due to Gauge symmetry, the amplitude $w_{1}$ can be assumed to be real and non-negative. The fixed points of the system are then obtained from the stationary solutions by setting $\lambda=0$.

\section{A. Stationary solutions and fixed points for the monomer}

For the monomer there is only one waveguide. For the stationary solution we obtain the algebraic set of equations

$$
\begin{aligned}
& -\lambda w_{1}+\chi w_{1} v_{1}=0, \\
& -(2 \lambda+\beta) v_{1}+\chi w_{1}^{2}=0,
\end{aligned}
$$

with $w_{1}^{2}+v_{1}^{2}=1$. Note that both amplitudes are real because $w_{1}$ is real. Bang et al. ${ }^{5}$ found stationary solutions for this system, which we summarize below.

\section{Solution MI}

The solution MI is obtained for $w_{1}=0$. This requires that $\lambda=-\beta / 2$ and $\left|v_{1}\right|^{2}=1$. No solution exists for $\lambda=0$, because $\beta=\pi$ is nonzero and thus we have no fixed points for the solution MI.

\section{Solution MII}

The solution MII exists for $v_{1}=\lambda / \chi$ and $w_{1}^{2}=\lambda(2 \lambda$ $+\beta) / \chi^{2}$. From the requirement that $w_{1}^{2}+v_{1}^{2}=1$ we find that the stationary solution MII exists for $3 \lambda^{2}+\lambda \beta-\chi^{2}=0$.

Setting $\lambda=0$ implies that $v_{1}=w_{1}=0$ could be a fixed point solution for $\chi=0$. However, due to the normalization with the total intensity $I$, the zero-solution is not a valid solution, so no fixed points occur for the type MII solutions.

For more details on the existence and linear stability of the solutions MI and MII, we refer to Bang et al. ${ }^{5}$

\section{B. Stationary solutions and fixed points for the dimer}

Let us now consider the dimer, or two-core coupler, for which $\rho \neq 0$. The stationary solutions for the dimer are obtained from the algebraic set of equations

$$
\begin{aligned}
& -\lambda w_{1}+\rho w_{2}+\chi w_{1} v_{1}=0, \\
& -(2 \lambda+\beta) v_{1}+\chi w_{1}^{2}=0, \\
& -\lambda w_{2}+\rho w_{1}+\chi w_{2} v_{2}=0, \\
& -(2 \lambda+\beta) v_{2}+\chi w_{2}^{2}=0,
\end{aligned}
$$

with $w_{1}^{2}+v_{1}^{2}+w_{2}^{2}+v_{2}^{2}=1$. Note that all amplitudes are real because $w_{1}$ is real. Bang et al. ${ }^{5}$ found stationary solutions for the more general dimer, or two-core coupler, where the sec- 
ond harmonics were also linearly coupled to each other. Setting this SH coupling to zero gives us the stationary solutions for our equations, which we summarize below.

\section{Solution DI}

For $\lambda=-\beta / 2=-\pi / 2$ the dimer solution DI exists. For this solution, $w_{n}=0$ and $v_{1}^{2}+v_{2}^{2}=1$. Because $\lambda \neq 0$ we again have no fixed point solutions with $\lambda=0$.

\section{Solution DII}

For $\lambda \neq-\beta / 2$, two more dimer solutions exist, both with $w_{n} \neq 0$ and $v_{n}=\chi w_{n}^{2} /(2 \lambda+\beta)$. The dimer solution DII has $w_{1}^{2}=w_{2}^{2}$, which means that it has two branches: $w_{2}= \pm w_{1}$ and $w_{1}>0$. This solution has the fundamental $w_{1}^{2}=(2 \lambda+\beta)$ $\times(-\lambda \pm \rho) / \chi^{2}>0$ and exists for $(\lambda+\pi / 2)(-\lambda \pm \rho)>0$. Setting $\lambda=0$, we find the fixed point solution $w_{1}^{2}= \pm \beta \rho / \chi^{2}>0$, $w_{2}= \pm w_{1}$, and $v_{n}= \pm \rho / \chi$.

\section{Solution DIII}

The dimer solution DIII also exists for $\lambda \neq-\beta / 2$ with $w_{n} \neq 0$ and $v_{n}=\chi w_{n}^{2} /(2 \lambda+\beta)$. However, in contrast to DII, the DIII solution is more general with $w_{2}^{2} \neq w_{1}^{2}$ and $w_{1}>0$. If we define the parameter $\alpha=\chi^{2} /(2 \lambda+\beta)$, then the DIII solution is given by $w_{1} w_{2}=\rho / \alpha$ with the fundamental $w_{1}=\sqrt{x}$ $>0$ being determined by the quadratic equation $\alpha^{2} x^{2}-\lambda \alpha x$ $+\rho^{2}=0$.

Setting $\lambda=0$, we find that $\alpha=\chi^{2} / \beta=\chi^{2} / \pi$ and $\alpha^{2} x^{2}+\rho^{2}$ $=0$. Since $\rho^{2} \geq 0$ and we must have that $x^{2}=w_{1}^{4}>0$, fixed point solutions only exist in the special linear case with $\chi=0$.

For more details on the existence linear stability of the solutions DI, DII, and DIII, we refer to Bang et al. ${ }^{5}$

\section{THE BREAK-UP OF THE LAST BARRIER PREVENTING $\left|W_{1}\right|^{2}=0$}

We now consider the dynamical evolution of the fields in the two-core coupler from the initial condition in which all intensity is in the FW, i.e., $W_{1 R}(\tau=0)=1$ with all seven other fields being zero at $\tau=0$. This so-called unseeded initial condition is typical in optical experiments. Our numerical simulations show that as $\rho$ is slowly increased from zero, for fixed values of $\beta=\pi$ and $\chi$, we move from a state at $\rho=0$, which is completely integrable and the phase space is foliated with invariant tori, to a state where none of the invariant tori exist any longer, and hence the transport is no longer confined to (or bounded by) barriers formed from invariant tori. This is important as the state with no barriers is a state where it is possible for the system to evolve from initially having all the input in one waveguide and in one of the variables (i.e., $\left.W_{1 R}=1\right)$ to, for example, having all its output in the second waveguide. Figures 3, 6, 7, and 9 have all been produced using DSTOOL. ${ }^{26}$

\section{A. The geometry and break-up of the barrier in $\left(W_{1 R}, W_{11}\right)$ space with changing $\rho$}

We study here what happens to the barrier seen in the $\left(W_{1 R}, W_{1 I}\right)$ part of the phase space as the parameters are changed. Due to our normalization, $\beta=\pi$ is fixed. We further fix the nonlinearity to $\chi=10$. We then vary $\rho$ from 0 to $\rho_{c}$ and above, where $\rho_{c}$ is the critical value of $\rho$, at which we break through the last barrier to global transport in $\left(W_{1 R}, W_{1 I}\right)$ space. In particular we look at the inner radius of the torus (when it exists) for different values of $\rho$. Conclusions are made regarding the way in which the orbits evolve on the torus; i.e., whether they have an irrational rotation number, in what direction do they wind around the tori, is the rotation clockwise or anticlockwise, or is the rotation number rational.

For $\rho=0$, it can be seen in Fig. 3(a) that the orbit winds around the torus in a quasiperiodic, clockwise manner until it densely covers the torus. This is expected as the system is completely integrable for $\rho=0$ and hence we expect the phase space to be foliated with such tori. We now perturb this integrable system (i.e., the two uncoupled monomers) by increasing the value of $\rho$ away from zero and towards $\rho_{c}$.

Increasing $\rho$ to 0.45 [see Fig. 3(b)], we begin to see that the outer limit of the torus is no longer so regular-it now has a quasiperiodic wavy shape. Note that the orbit is still winding around the torus in a quasiperiodic clockwise manner.

If we now increase $\rho$ to 0.55 [see Fig. 3(c)], we find that the orbit winds around the torus much slower; this means that the rotation number is very close to being rational. As a result the orbit almost returns to the same position after a certain time period. Note, however, that the orbit is still winding round the torus in a clockwise manner.

A further increase in $\rho$ to 0.65 shows a major change [see Fig. 3(f)]. The orbits are still bound by a torus and are winding around the torus in a quasi periodic manner, however in an anticlockwise direction.

This means that for some value $\rho_{r}$ between $\rho=0.55$ and $\rho=0.65$ we get a rational rotation number. See Figs. 3(d) and 3(e) for the second harmonic in the first waveguide for $\rho$ $=0.58$. A close look at these figures shows, however, that the orbit is only approximately periodic. The reason is that the amplitudes of both harmonics in the second waveguide are small but not of the same period as in the first waveguide. Such approximate periodicity suggests the presence of a resonant region nearby in the phase space, the existence of which could be shown analytically using normal forms. ${ }^{8}$ For resonant or periodic conditions to be observed in the $\left(W_{1 R}, W_{1 I}\right)$ space, the amplitudes of all the other waves would either have to be zero or be periodic and of the same period.

Further increases in $\rho$ lead to the destruction of the torus, which binds the motion [see Fig. $3(\mathrm{~g})]$. We see that the barrier appears to remain a barrier for a long period of time until eventually the orbit crosses the barrier and evolves in a different region of the phase space. These barriers, which appear to be complete (i.e., no orbits are allowed through, for our specific initial conditions and for $\tau \leq \tau_{0}$ ) but which in fact are not (i.e., the orbit corresponding to our specific ini- 


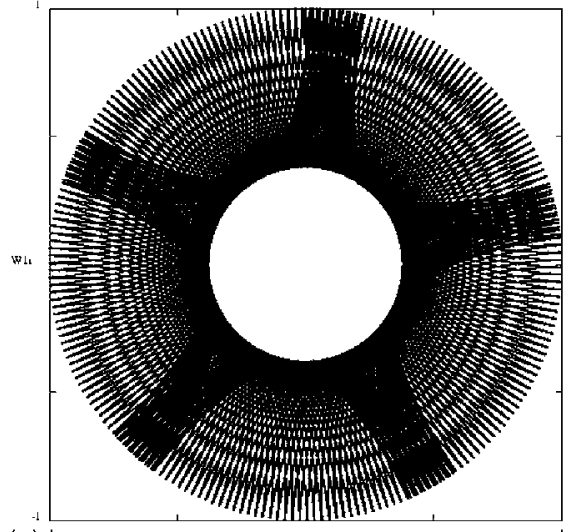

(a)

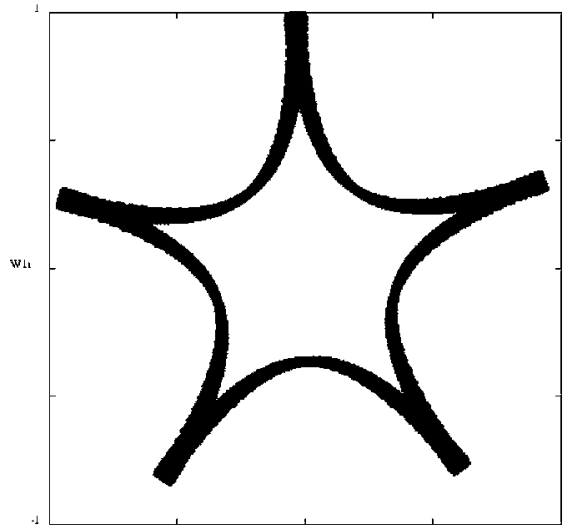

(d)

w!

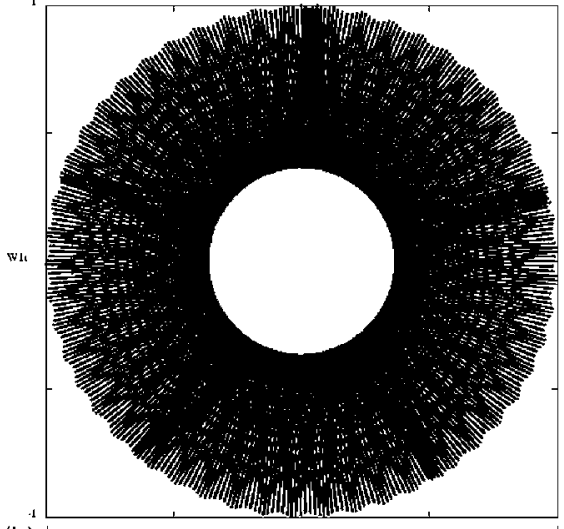

(b)

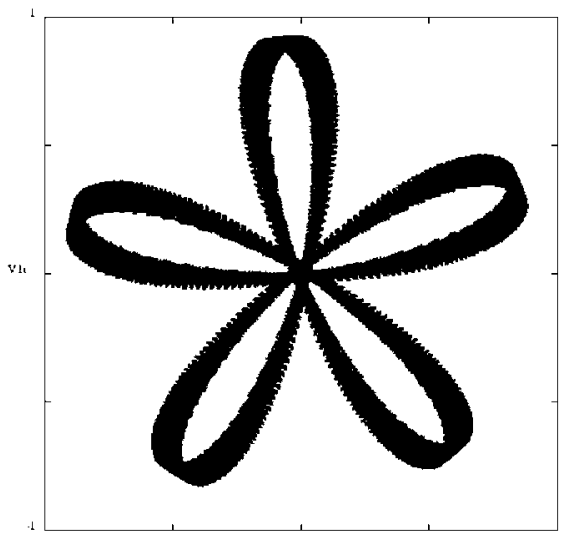

(e)

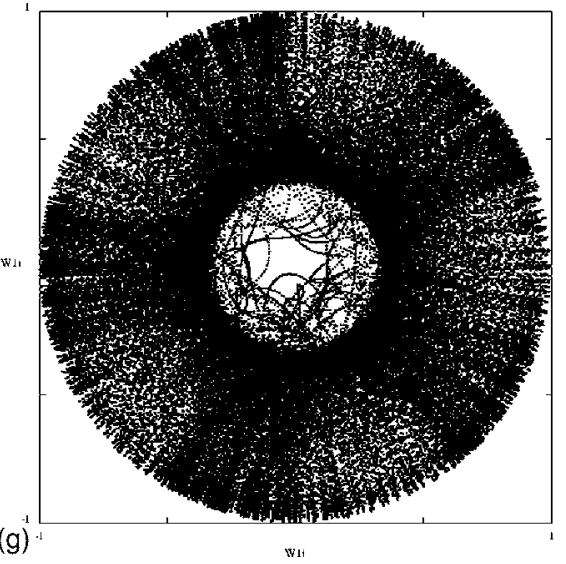

$\mathrm{vi}$

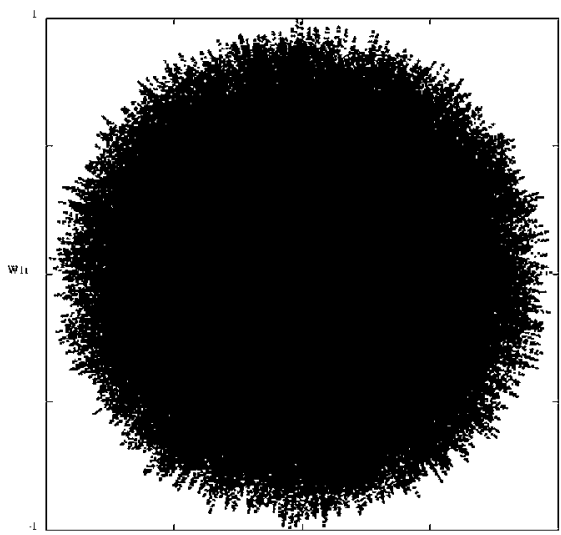

$(h)^{-1}$

(n)

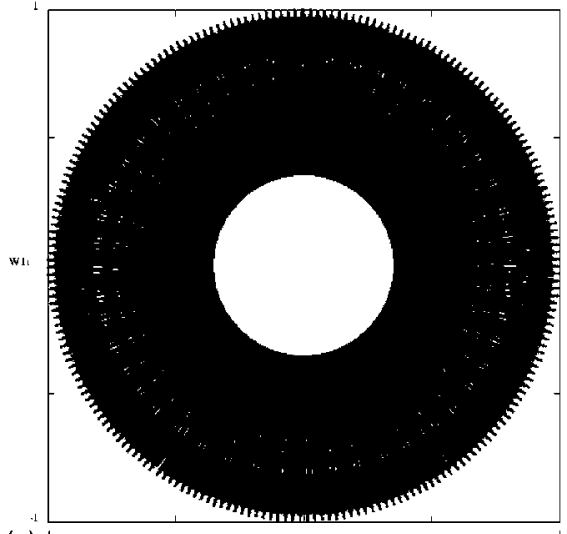

(c)

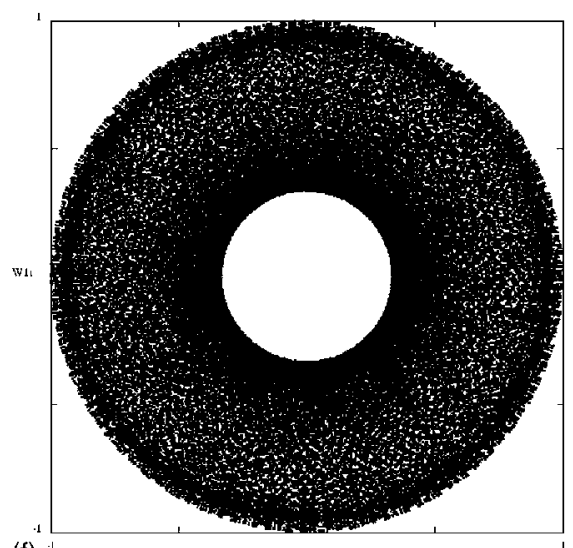

(f)

wli

FIG. 3. For all axes, $-1 \leq W_{1 R}, W_{1 I}, V_{1 R}, V_{1 I} \leq 1$. (a) $W_{1 R}$ vs $W_{1 I}$ for $\chi=10, \beta=\pi$, and $\rho=0$; (b) $W_{1 R}$ vs $W_{1 I}$ for $\chi=10, \beta=\pi$, and $\rho=0.45$; (c) $W_{1 R}$ vs $W_{1 I}$ for $\chi=10, \beta=\pi$, and $\rho=0.55$; (d) $W_{1 R}$ vs $W_{1 I}$ for $\chi=10, \beta=\pi$, and $\rho=0.58$; (e) $V_{1 R}$ vs $V_{1 I}$ for $\chi=10, \beta=\pi$, and $\rho=0.58$; (f) $W_{1 R}$ vs $W_{1 I}$ for $\chi=10$, $\beta=\pi$, and $\rho=0.65$; $(\mathrm{g}) W_{1 R}$ vs $W_{1 I}$ for $\chi=10, \beta=\pi$, and $\rho=0.68$; and (h) $W_{1 R}$ vs $W_{1 I}$ for $\chi=10, \beta=\pi$, and $\rho=0.69$.

tial conditions eventually crosses the barrier for $\tau \leq \tau_{0}$ ), will be termed partial barriers. These partial barriers could be due to nearby "sticky" Kolmogorov-Arnold-Moser (KAM) tori. Note that in using this definition, some of the barriers we define as complete could in fact be partial for different initial conditions and for values of $\tau$ greater than $\tau_{0}$. However, we use these definitions as we are interested in what would be observed practically, and hence we are only interested in our specific initial conditions and the range of $0 \leq \tau \leq \tau_{0}$. Partial barriers are important, because if the likelihood of transport across them is sufficiently small, then practically speaking, they act as though they are complete. It is observed that the trajectory is now more complicated and is no longer quasiperiodic. Instead, the trajectory evolves around the torus in such a manner that we can observe many changes in the direction of rotation from clockwise to anticlockwise and back again (unlike in the case before, where the orbit just rotated in one direction) as the orbit densely covers the surface. Note that Figs. 3(d) $-3(\mathrm{~g})$ are all drawn for the same range of $\tau$; i.e., the same $\tau_{0}$.

If we increase $\rho$ further still (i.e., to $\rho=0.69$ ), we then see that the partial barrier has a minimal effect on the dynamics on the time scales that we are interested in and hence we do not feel its effect significantly. As expected, this shows 


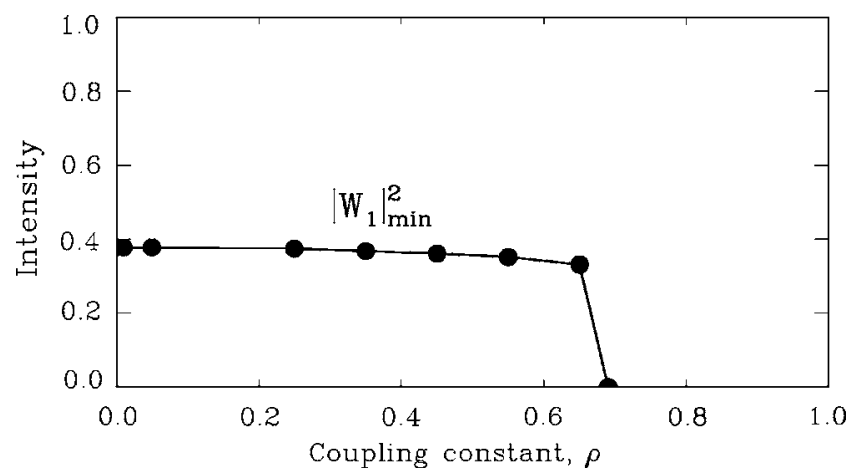

FIG. 4. $\left|W_{1}\right|_{\min }^{2}$ vs $\rho$ for $\chi=10, \beta=\pi$. Notice that $\rho_{c}$ is the minimum value of $\rho$ for which $\left|W_{1}\right|_{\min }^{2}=0$.

that the rate of transport across these barriers is a function of $\rho$ (when $\rho<\rho_{c}$, we get no transport across them, but for $\rho$ $>\rho_{c}$ in the ranges we have considered, the rate of transport across increases with $\rho$ ). This behavior is important as it indicates the kind of values of $\rho$ that are needed for fixed values of $\chi$ and $\beta$ to obtain an chaotic transfer of intensity from the fundamental harmonic in the first waveguide to either of the harmonics in the second waveguide.

We also measured the inner radius of the torus for different values of $\rho$. This gives a value for the minimum of the intensity in the fundamental wave; i.e., a value for $\left|W_{1}\right|_{\text {min }}^{2}$ (see Fig. 4). For this figure, it should be remembered that, for $\rho>\rho_{c}$, the circle no longer exists; neither does it exist when the orbit is in resonance, i.e., $\rho=0.58$.

The values of $\rho_{c}$ for a range of $\chi$ from 1 to 20 and $\beta$ $=\pi$ are also calculated. As can be seen in Fig. 5, there is an approximately linear relationship between $\rho_{c}$ and $\chi$ for the range of parameter values we considered. It was also noticed that when $\rho>\rho_{c}$, lower values of $\chi$ give a wave with a complicated amplitude changing more slowly and having less disorder than a wave that is calculated for higher values of $\chi$.

We also found approximate values for $\rho_{r}$ (the value of $\rho$ for which we get resonance) to be $0.2725,0.471$, and 0.58 for $\chi$ equal to 4,7 , and 10 , respectively. It should be noted, however, that the rotation number was different in each case, but we could still observe the same type of phenomena we described for $\chi=10$.

We investigate now the possible mechanism for the destruction of the complete barrier. It can be shown for systems evolving on the plane that, as the perturbation of the inte-

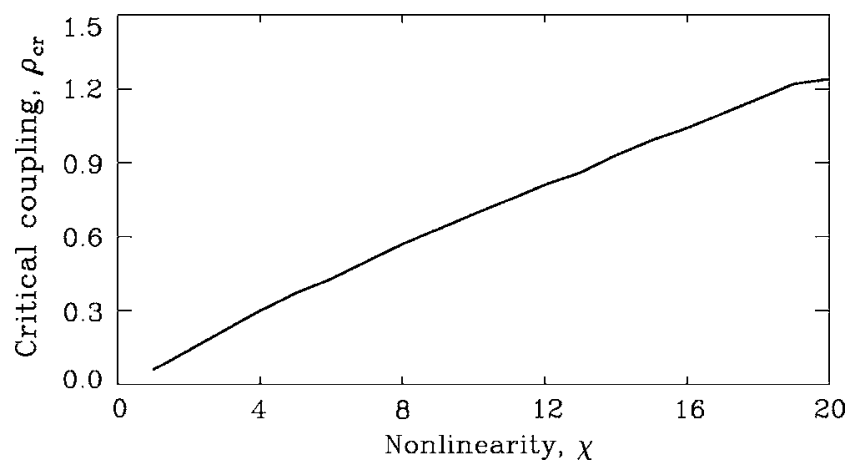

FIG. 5. $\rho_{c}$ vs $\chi$, for $\beta=\pi$. grable case is increased, the resonance appears and grows in size, and the region of the phase space occupied by the invariant tori shrinks. It is also found that invariant tori are destroyed when resonances engulfs the region of phase space they once occupied (see Meiss ${ }^{13}$ ). In our system it appears that the orbit gets trapped in a resonance zone in between the case when it evolves on a torus and the case where there is global transport in $W_{1}$ space. This can be seen by the existence of particular values of $\rho$ for which we have a change in the way of rotation around the torus, and also in the fact that after this occurs (i.e., for $\rho_{r}<\rho<\rho_{c}$ ), we have a motion that is more irregular than before (but not as irregular as for values of $\rho \geq \rho_{c}$ ), and which appears to be bound between two tori, up until $\rho \geq \rho_{c}$. This is consistent with what happens for systems that evolve on the plane as we increase the perturbation.

\section{B. Partial and complete barriers and their effect on how the intensity $\left|W_{1}\right|^{2}$ changes with time}

We now study how the presence or absence of partial or complete barriers affects the intensity of the fundamental and second harmonics in each waveguide. We do this by investigating how the intensities varies with time and with each one of the cases where we have a complete barrier, an approximate resonance, a partial barrier, and finally no barrier of any consequence.

The first case studied [see Fig. 6(a)] is the case of the complete barrier. As an example, we take $\rho=0.65, \chi=10$, and $\beta=\pi$. This corresponds to Fig. 3(f). As we can see from Fig. $6(\mathrm{a})$, the intensity in the fundamental remains bound by an upper $\left(\left|W_{1}\right|^{2}=1\right)$ and lower value $\left(\left|W_{1}\right|^{2}=\left|W_{1}\right|_{\text {min }}^{2}\right)$, where $\left|W_{1}\right|_{\text {min }}^{2}$ is a constant corresponding to the inner radius of the tori seen in Fig. 3(f).

We now increase the value of $\rho$ to 0.68 [see Fig. 6(b)] to show the effect of the partial barrier seen in Fig. 3(g).

As can be seen in Fig. 6(b), the intensity appears bound for quite a period of time but, when the orbit breaks through the partial barrier, a dramatic change in the minimum value of the intensity $\left|W_{1}\right|^{2}$ is observed; i.e., its minimum now becomes 0 and the intensity no longer oscillates between $\left|W_{1}\right|_{\text {min }}^{2}$ and 1 .

We now increase the value of $\rho$ to 0.69 [see Fig. 6(c)] to show the effect of the lack of a presence of a barrier of any consequence for the minimum intensity $\left|W_{1}\right|_{\min }^{2}$, as can be seen in Fig. 3(h). The amplitude here is characteristic of that resulting from a relatively high dimensional system in which the orbit is evolving in a spatially temporally chaotic manner.

Finally we study the case where the orbit is nearly resonant [see Fig. 6(d)], which corresponds to Figs. 3(d) and $3(\mathrm{e})$. We notice that the minimum and maximum values are no longer a constant but vary in time in some periodic manner. This is to be expected by observation of Figs. 3(d) and $3(\mathrm{e})$ : the orbits are nearly resonant. 

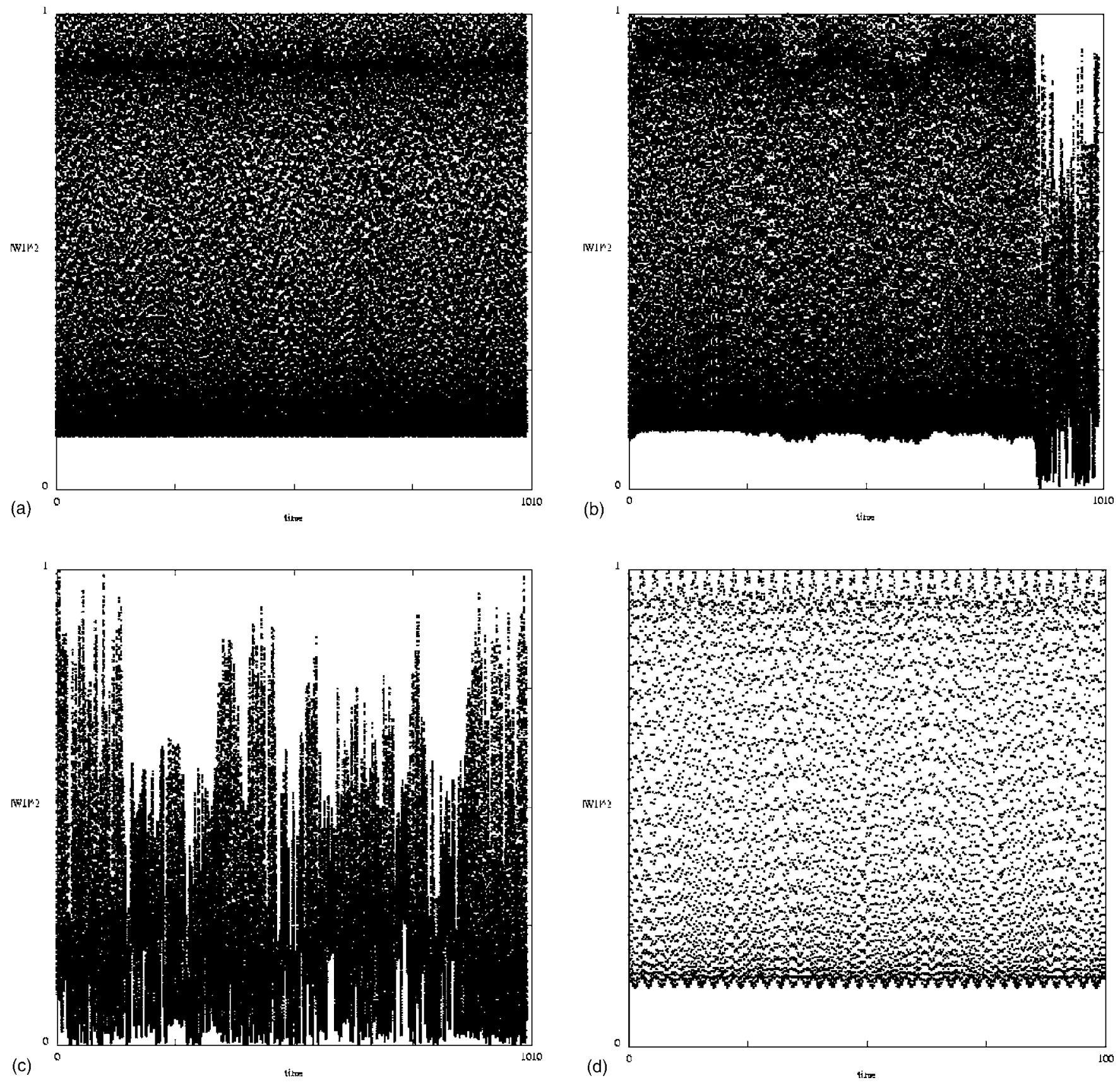

FIG. 6. The intensity $\left|W_{1}\right|^{2}$ vs time for different values of $\rho$ with fixed $\beta=\pi$ and $\chi=10$. On all axes, $0 \leq\left|W_{1}\right|^{2} \leq 1$ and time runs from $\tau=0$ to $\tau=1010$. (a) $\rho=0.65$; (b) $\rho=0.68$; (c) $\rho=0.69$; and (d) $\rho=0.58$.

\section{Partial and complete barriers and the relationship between the intensity in each of the harmonics}

Having studied how the intensity varies with time, we now concentrate on investigating how the intensity $\left|W_{1}\right|^{2}$ varies with $\left|W_{2}\right|^{2}$ and $\left|V_{2}\right|^{2}$. This will show how the intensity is transferred between the waves in the two different waveguides. We follow the same procedure as before and study the case where we have complete and partial barriers as well as the case where there is no barrier of any consequence and the case when the orbit is close to a resonance.

We start with the case where there is a full barrier; i.e., $\rho=0.65$. We then increase $\rho$ to 0.68 and finally up to $\rho=0.69$. For Fig. 7, it should be noticed that the intensities of $W_{1}$ and $W_{2}$ are never simultaneously zero. This explains the fact that the intensity is never equal to 1 for $\left|V_{1}\right|^{2}+\left|V_{2}\right|^{2}$ for the parameter ranges we consider. This implies the existence of other barriers to motion in phase space, which prevent $\left|V_{1}\right|+\left|V_{2}\right|^{2}$ from becoming 1 , even when $\left|W_{1}\right|^{2}$ becomes 0 . It can also be seen in Fig. 8 that, for values of $\rho$ of approximately 1 or less, we have a constant minimum intensity that is the same as that existing for $\rho=0$. We also observe that $\left|W_{1}\right|_{\min }^{2}+\left|W_{2}\right|_{\min }^{2}=C_{1}$, where $C_{1}$ is a constant, whereas for larger values of $\rho$, we have the relationship $\left|W_{1}\right|_{\min }^{2}+\left|W_{2}\right|_{\text {min }}^{2}$ $=C_{2}$, where $\left|W_{1}\right|_{\min }^{2}+\left|W_{2}\right|_{\min }^{2}=1$ for $\rho$ so large that $\chi$ can be 

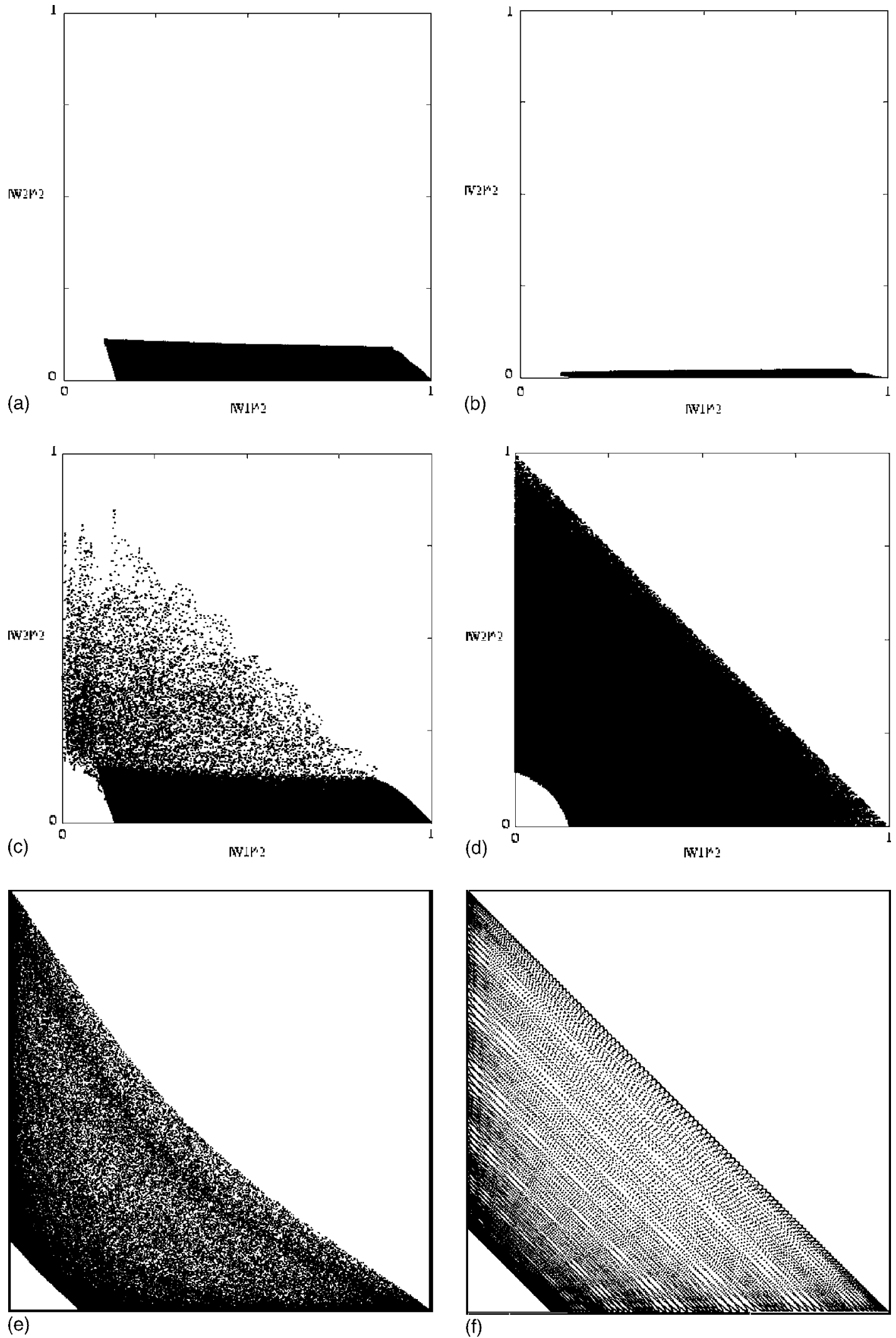

FIG. 7. Intensity evolution for different values of $\rho$ with fixed $\beta=\pi$ and $\chi=10$. On all axes, $0 \leq\left|W_{1}\right|^{2},\left|W_{2}\right|^{2},\left|V_{2}\right|^{2} \leq 1$. (a) $\left|W_{1}\right|^{2}$ vs $\left|W_{2}\right|^{2}$ for $\rho=0.65$; (b) $\left|W_{1}\right|^{2}$ vs $\left|V_{2}\right|^{2}$ for $\rho=0.65$; (c) $\left|W_{1}\right|^{2}$ vs $\left|W_{2}\right|^{2}$ for $\rho=0.68$; (d) $\left|W_{1}\right|^{2}$ vs $\left|W_{2}\right|^{2}$ for $\rho=0.69$; (e) $\left|W_{1}\right|^{2}$ vs $\left|W_{2}\right|^{2}$ for $\rho=10$; and $\left|W_{1}\right|^{2}$ vs $\left|W_{2}\right|^{2}$ for $\rho=100$.

considered effectively equal to zero. It should also be noted that, by observation of how the trajectory evolves in Fig. 7(c), partial barriers can be found to exist in the phase space for $\rho=0.68$. This can be easily seen from the density of the points in these figures.
Figure 8 shows how the minimum of $\left|W_{1}\right|^{2}+\left|W_{2}\right|^{2}$ varies with $\rho$ for $\chi=10$ and $\beta=\pi$. It can be seen that for small values of $\rho,\left(\left|W_{1}\right|^{2}+\left|W_{2}\right|^{2}\right)_{\min }$ remains constant, having the same value as the ones that can be obtained from the integrable case, where $\rho=0$. This means it may be possible to 


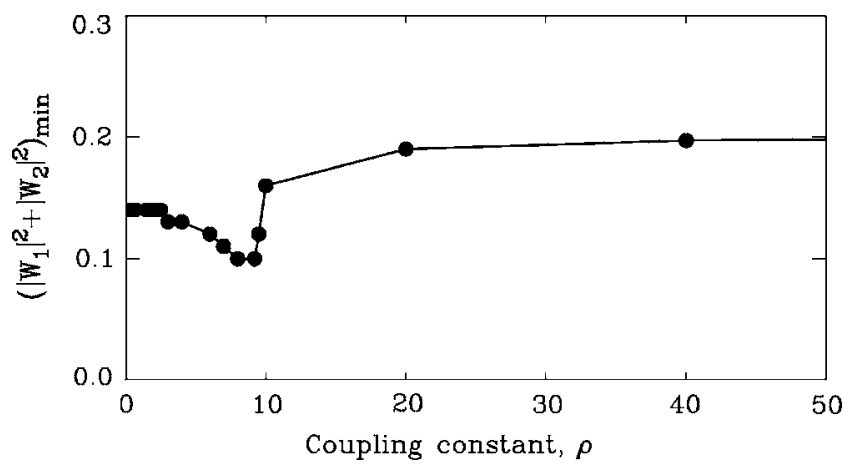

FIG. 8. $\left(\left|W_{1}\right|^{2}+\left|W_{2}\right|^{2}\right)_{\min }$ vs $\rho$, for $\chi=10$ and $\beta=\pi$. Note that $\left(\left|W_{1}\right|^{2}\right.$ $\left.+\left|W_{2}\right|^{2}\right)_{\text {min }}$ remains equal to 0.2 for values of $\rho$ up to 1000 .

obtain an analytical solution for this value. At the other limit, we know that, as $\rho$ becomes infinitely larger than $\chi$, then $\left(\left|W_{1}\right|^{2}+\left|W_{2}\right|^{2}\right)=1$ for all time, as no intensity is transferred to $V_{n}$ (see Fig. 9).

\section{CONCLUSIONS}

The present study shows the importance of barriers to transport in the phase space of the model (1) and (2) of two coupled quadratic nonlinear waveguides, and the effect the barriers have on the transfer of intensity between waveguides. This is important as the parameter values for which the last barrier to global transport in $W_{1}$ space is broken provide the minimum value for which there is complete transference of intensity from the fundamental in the first waveguide $\left(\left|W_{1}\right|^{2}\right)$ to the other harmonics. Figure 4 shows the presence of a critical coupling strength $\rho_{c}$, which is the minimum value of $\rho$ for which $\left|W_{1}\right|^{2}$ can go from initially

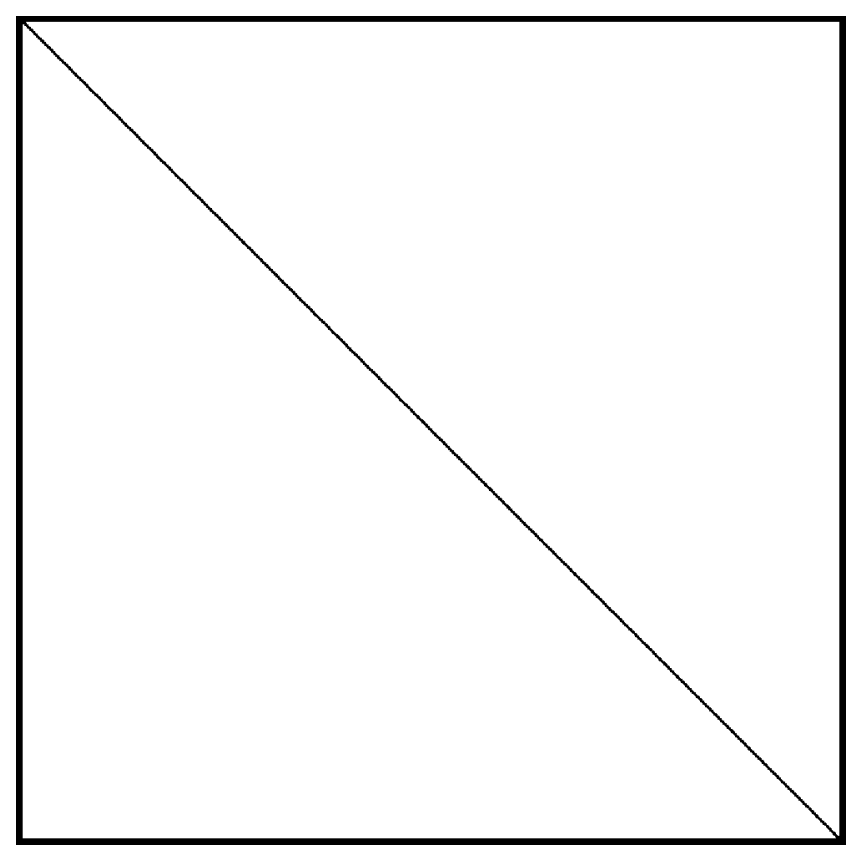

FIG. 9. The intensity $\left|W_{1}\right|^{2}$ vs $\left|W_{2}\right|^{2}$ for $\chi=0.001, \beta=\pi$, and $\rho=100$. On the axes, $0 \leq\left|W_{1}\right|^{2},\left|W_{2}\right|^{2} \leq 1$. Note that this is effectively the same as taking $\rho$ very large for $\chi=10$. being equal to 1 to being equal to 0 after a finite time. Figure 5 shows the values of $\rho_{c}$ for different values of $\chi$, keeping $\beta=\pi$.

We also show that, just before the last barrier preventing $\left|W_{1}\right|^{2}=0$ breaks up, the orbit gets trapped near a resonance zone. We make the conjecture that the barrier breaks up because it becomes engulfed by a resonance. We show that partial barriers in the phase space also exist. These partial barriers are important as they act as complete barriers for long time periods and then suddenly the orbit breaks through them and is able to explore more of the phase space. What is of interest, practically speaking, is that the partial and complete barriers can be almost indistinguishable for long time periods, depending on the initial conditions and on how easy it is to cross the partial barrier. We show that the intensity $\left|W_{1}\right|^{2}$ becomes very disordered (i.e., it appears spatially and temporally chaotic) for a very small increase in $\rho$ past $\rho_{c}$ (for $\rho<\rho_{c}$ the orbit evolves on a torus and hence the intensity is trapped between an upper and lower limit). It is important to note the disordered pattern of the intensity in time as it shows that such responses are inherent in the system and are not just a product of noise or experimental error. What is also important is the proximity, in terms of the coupling $\rho$, between the ordered and disordered response.

It is also shown that the barrier, which prevents the transport in $\left|W_{1}\right|$ space, is not the last barrier to break down in the phase space in the range of parameter values we looked at ( $\rho$ variable, $\chi=10$, and $\beta=\pi$ ). This can be seen in Figs. 7(c) and 7(d), in which we can see that we never obtain the intensities $\left|W_{1}\right|^{2}=\left|W_{2}\right|^{2}=0$. Figure 8 shows how $\left(\left|W_{1}\right|^{2}\right.$ $\left.+\left|W_{2}\right|^{2}\right)_{\min }$ varies with the coupling constant $\rho$. It can be seen that for $\rho$ small, this is almost a constant of similar size as for $\rho=0$, whereas for $20 \leq \rho \leq 1000,\left(\left|W_{1}\right|^{2}+\left|W_{2}\right|^{2}\right)_{\text {min }}$ is again approximately constant, but is now equal to 0.2 . This is important as it shows that if we want to find the minimum value, which transfers all the intensity to being in either of the second harmonics, we have to investigate the break-up of some of the other barriers in the phase space.

Finally, we note that we can also obtain interesting phenomena by perturbing the integrable case of $\rho \neq 0, \chi=0$ with small values of $\chi$. This would be a study of a route to chaos from a coupling limit other than the one we have just studied. It should also be noted that further interesting information regarding the location of resonances and close invariant tori in the part of the phase space relevant to this application could be provided with the use of geometrical and analytical tools, such as normal forms and KAM theory.

\section{ACKNOWLEDGMENTS}

This work was partially supported by the programs Ramón y Cajal 2004 and I3 2006, Ministerio de Educación y Ciencia, Spain.

${ }^{1}$ V. M. Agranovich and J. B. Page, "Fermi resonance interface modes and bistable energy transmission through the interface," Phys. Lett. A $\mathbf{1 8 3}$ 395 (1993).

${ }^{2}$ S. A. Dubovskii and A. V. Orlov, "New type of nonlinear interface waves under the conditions of Fermi resonance of the vibrations of adjoining crystals," Fiz. Tverd. Tela (Leningrad) 38, 1221 (1996) [Phys. Solid State 38, 675 (1996)] 
${ }^{3}$ T. Peschel, U. Peschel, and F. Lederer, "Solitary waves in Bragg gratings with a quadratic nonlinearity," Phys. Rev. E 55, 4730 (1997).

${ }^{4}$ P. D. Miller and O. Bang, "Macroscopic dynamics in quadratic nonlinear lattices," Phys. Rev. E 57, 6038 (1998).

${ }^{5}$ O. Bang, P. L. Christiansen, and C. B. Clausen, "Stationary solutions and self trapping in discrete quadratic nonlinear systems," Phys. Rev. E 56, 7257 (1997).

${ }^{6}$ V. I. Arnold, Mathematical Methods of Classical Mechanics, in Graduate Texts in Mathematics (Springer-Verlag, Berlin, 1989).

${ }^{7}$ G. Haller and S. Wiggins, "Geometry and chaos near resonant equilibria of 3-DOF Hamiltonian systems," Physica D 90, 319 (1996).

${ }^{8}$ G. Haller, Chaos Near Resonance, Applied Mathematical Sciences (Springer, New York, 1999), Vol. 138.

${ }^{9}$ A. J. Lichtenberg and M. A. Lieberman, Regular and Chaotic Dynamics, Applied Mathematical Sciences (Springer, Berlin, 1992), Vol. 138.

${ }^{10} \mathrm{~A}$. Litvak-Hinenzon and V. Rom-Kedar, "Resonant tori and instabilities in Hamiltonian systems," Nonlinearity 15, 1149 (2002).

${ }^{11}$ A. Litvak-Hinenzon and V. Rom-Kedar, "On energy surfaces and the resonance web," SIAM J. Appl. Dyn. Syst. 3, 525 (2004).

${ }^{12}$ A. Litvak-Hinenzon and V. Rom-Kedar, "Parabolic resonances in 3 d.o.f. near integrable Hamiltonian systems," Physica D 164, 213 (2004).

${ }^{13}$ J. D. Meiss, "Symplectic maps, variational principles, and transport," Rev. Mod. Phys. 64, 795 (1992).

${ }^{14}$ V. Rom-Kedar and S. Wiggins, "Transport in two-dimensional maps," Arch. Ration. Mech. Anal. 109, 239 (1990).

${ }^{15} \mathrm{~V}$. Rom-Kedar, "Transport in a class of n-d.o.f. systems," in Hamiltonian Systems with Three or More Degrees of Freedom (S'Agar'o, 1995) (Kluwer Academic, Dordrecht, 1999), pp. 538-543.

${ }^{16} \mathrm{~S}$. Wiggins, "On the geometry of transport in phase space 1. Transport in a k-degree-of-freedom Hamiltonian system, $2 \leq k \leq \infty$," Physica D 44, 471 (1990)
${ }^{17}$ S. Wiggins, Chaotic Transport in Dynamical Systems, Interdisciplinary Applied Mathematics (Springer-Verlag, Berlin, 1992).

${ }^{18}$ G. M. Zaslavsky, R. Z. Sagdeev, D. A. Usikov, and A. A. Chernkow, Weak Chaos and Quasi-Regular Patterns, Cambridge Nonlinear Science (Cambridge University Press, Cambridge, 1991), Vol. 1.

${ }^{19}$ A. Delshams, R. de la Llave, and T. M. Seara, "Orbits of unbounded energy in quasi-periodic perturbations of geodesic flows," Adv. Math. 202, 64 (2006).

${ }^{20}$ A. Delshams, R. de la Llave, and T. M. Seara, "Geometric mechanism for diffusion in Hamiltonian systems overcoming the large gap problem: Heuristics and rigorous verification on a model," Mem. Am. Math. Soc. 179, 1 (2006).

${ }^{21}$ A. Delshams, R. de la Llave, and T. M. Seara, "A geometric approach to the existence of orbits with unbounded energy in generic periodic perturbations by a potential of generic geodesic flows of the torus," Commun. Math. Phys. 209, 353 (2000).

${ }^{22}$ J. M. Ottino, The Kinematics of Mixing: Stretching, Chaos, and Transport, Cambridge Texts in Applied Mathematics (Cambridge University Press, Cambridge, 1989).

${ }^{23}$ J. R. Stirling, "Dynamical systems models of patchiness in estuaries," Ph.D. thesis, Department of Mathematical Sciences, Loughborough University, UK, 1999.

${ }^{24}$ J. R. Stirling, "Transport and bifurcation in a non area preserving 2 dimensional map with applications to the discharge of pollution in an estuarine flow," Physica D 144, 169 (2000).

${ }^{25}$ J. R. Stirling, "Nonlinear dynamics, transport and the patchiness of clouds of pollution in an estuarine fluid flow," Discrete Contin. Dyn. Syst., Ser. B 3, 263 (2003)

${ }^{26}$ A. Back, J. Guckenheimer, M. R. Myers, F. J. Wicklin, and P. A. Worfolk, "DsTool: Computer assisted exploration of dynamical systems," Not. Am. Math. Soc. 39, 303 (1992) 